\title{
NO-induced reversible switching of the electronic interaction between a porphyrin-coordinated cobalt ion and a silver surface
}

\author{
Ken Flechtner, Andreas Kretschmann, Hans-Peter Steinrück, and J. Michael Gottfried* \\ Universität Erlangen-Nürnberg, Lehrstuhl für Physikalische Chemie II, Egerlandstr. 3, \\ 91058 Erlangen, Germany
}

\section{Supporting Information}

\section{Experimental Details}

All experiments were performed with a commercial X-ray photoelectron spectrometer (Scienta ESCA$200)$ equipped with an Al- $\mathrm{K}_{\alpha} \mathrm{X}$-ray source $(1486.6 \mathrm{eV})$ with monochromator and a hemispherical energy analyzer (SES-200). The overall energy resolution of the spectrometer amounts to $0.3 \mathrm{eV}$. The base pressure in the UHV system is below $1 \times 10^{-10}$ mbar. The system also comprises a differentially pumped gas discharge lamp for UV photoelectron spectroscopy (UPS), LEED optics (ErLEED-1000A), two ion sources, a mass spectrometer (Balzers QMS 112), and various evaporators. CoTPP was evaporated with a home-built Knudsen cell evaporator at a temperature of $638 \mathrm{~K}$, measured at the quartz glass crucible. The resulting fluxes, typically $0.05 \AA / \mathrm{s}$ at the sample position, were measured with a quartz crystal microbalance. The sample was a discoidal Ag single crystal $(\varnothing 8 \mathrm{~mm}$, thickness $1.5 \mathrm{~mm}$, purity $>$ 99.999 \%, Surface Preparation Laboratory) with a polished (111) surface, which was aligned to $<0.1^{\circ}$ with respect to the nominal orientation. Two type $\mathrm{K}$ thermocouples were mounted directly to the rim of the Ag crystal for precise temperature measurement $( \pm 5 \mathrm{~K})$. The crystal was attached to a manipulator, which allows for cooling and heating in the temperature range $95 \mathrm{~K}-900 \mathrm{~K}$.

The UP spectra in Fig. 2 were taken with He-I radiation $(21.22 \mathrm{eV})$ and a sample bias of $-10 \mathrm{~V}$. The overall energy resolution for UPS was $<0.1 \mathrm{eV}$. The reported XPS and UPS binding energies are referenced to the Fermi edge of the clean $\mathrm{Ag}$ surface $\left(\mathrm{E}_{\mathrm{B}} \equiv 0\right)$. Photoemission detection geometries: Curves (C) and (D) in Fig. 1 were recorded in normal emission, curves (A) and (B) in grazing emission $\left(80^{\circ}\right.$ with respect to the surface normal) for improved signal-to-noise ratio. The UP spectra in Fig. 2 were taken in normal emission.

The tetraphenylporphyrinato-Co(II) (purity $>98 \%$, Porphyrin Systems GbR) was degassed in vacuo for $24 \mathrm{~h}$ at $420 \mathrm{~K}$ prior to the evaporation deposition.

The coverage $\theta$ is defined as the number of adsorbed molecules or atoms divided by the number of substrate atoms, whereas "monolayer" denotes a closed, saturated adlayer of molecules in direct contact with the substrate surface at $300 \mathrm{~K}$. Monolayer coverage corresponds to $\theta=0.037$. $^{\mathrm{S}}$

CoTPP monolayers were prepared by vapor deposition of CoTPP multilayers on the clean $\operatorname{Ag}(111)$ surface (see Ref. (S1) for details about sample preparation) followed by thermal desorption of the excessive multilayers at $550 \mathrm{~K}$ as explained in Ref. (S1). For the preparation of the NO-CoTPP monolayer, NO gas $(>99.5 \%$, Linde $A G)$ was dosed on a CoTPP monolayers with a pressure of $3 \times 10^{-8}$ mbar at a sample temperature of $140 \mathrm{~K}$. The total NO dosage was $300 \mathrm{~L}$ (Langmuir, $1 \mathrm{~L}=1 \times 10^{-6}$ Torr $\times \mathrm{s}$ ). NO-CoTPP multilayers were prepared by dosing NO $\left(3 \times 10^{-8} \mathrm{mbar}\right)$ during the vapor deposition CoTPP at $140 \mathrm{~K}$ (total NO dosage: $800 \mathrm{~L}$ ). The XP and UP measurements on NO-CoTPP were performed at $140 \mathrm{~K}$ 
at an NO background pressure of $3 \times 10^{-8}$ mbar. No adsorption of NO on the clean $\mathrm{Ag}(111)$ surface was observed under these conditions.

\section{References}

(S1) Lukasczyk, T.; Flechtner, K.; Merte, L. R.; Jux, N.; Maier, F.; Gottfried, J. M.; Steinrück, H. P. Journal of Physical Chemistry C 2007, 111, 3090-3098.

\section{Additional references concerning catalysis on supported metalloporphyrins}

(A1) Berner, S.; Biela, S.; Ledung, G.; Gogoll, A.; Backvall, J. E.; Puglia, C.; Oscarsson, S. Journal of Catalysis 2006, 244, 86-91.

(A2) Hassan, S. A.; Hassan, H. A.; Hashem, K. M.; Dayem, H. M. A. Applied Catalysis a-General 2006, $300,14-23$.

(A3) Ferreira, A. D. Q.; Vinhado, F. S.; Iamamoto, Y. Journal of Molecular Catalysis a-Chemical 2006, 243, 111-119.

\section{Supporting Figures}

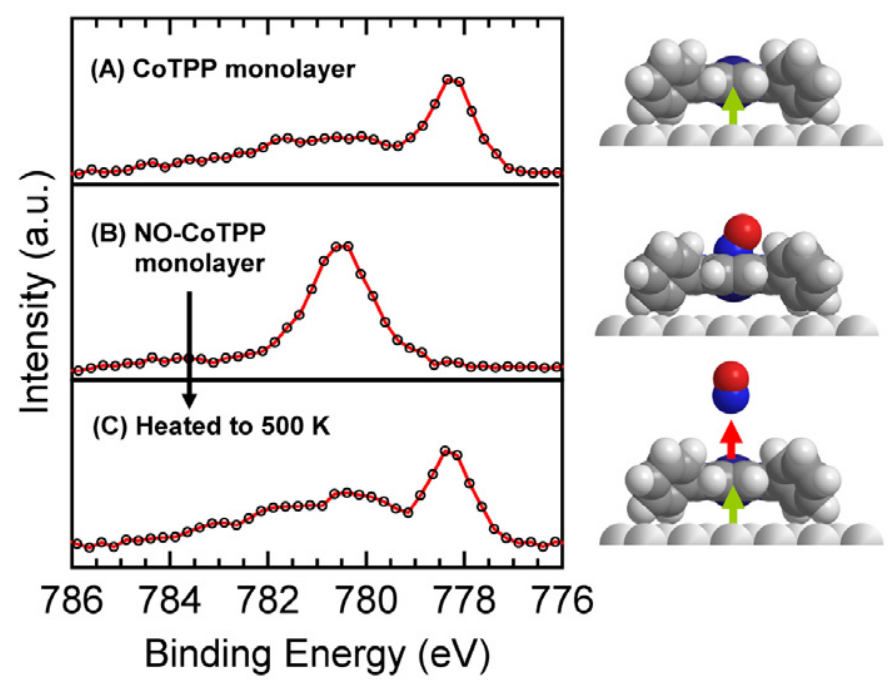

Figure S1: Co $2 \mathrm{p}_{3 / 2}$ XP spectra of CoTPP and NO-CoTPP on Ag(111). (A) CoTPP monolayer, (B) NOCoTPP monolayer, (C) after heating the NO-CoTPP monolayer to $500 \mathrm{~K}$ for removal of the NO ligand. All curves were recorded in grazing emission $\left(80^{\circ}\right.$ with respect to the surface normal) for improved signal-to-noise ratio. 


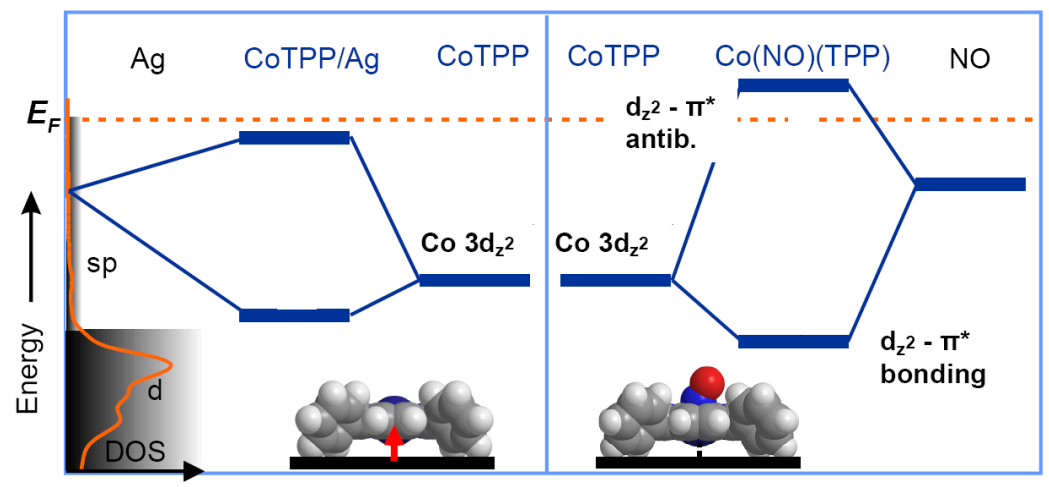

Figure S2: Molecular orbital (MO) model of the interaction of the half-occupied $3 \mathrm{~d}_{\mathrm{z}^{2}}$ orbital of the coordinated Co ion with the $\operatorname{Ag}(111)$ surface (left) and with the $\pi^{*}$ orbital of NO (right).

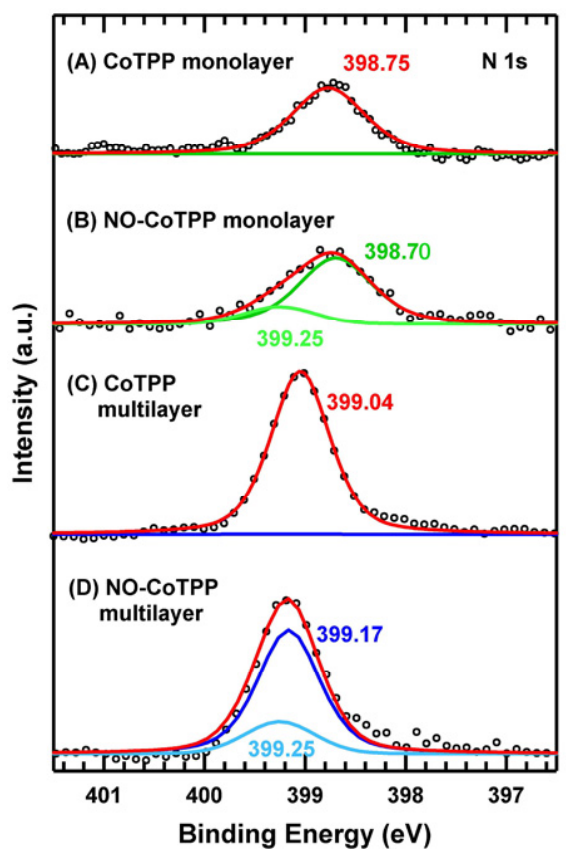

Figure S3: N 1s XP spectra of CoTPP and NO-CoTPP on Ag(111). (A) CoTPP monolayer, (B) NOCoTPP monolayer, (C) CoTPP multilayer (ca. 4 monolayers), (D) NO-CoTPP multilayer (ca. 4 monolayers). The spectra were recorded in grazing emission $\left(80^{\circ}\right.$ with respect to the surface normal). The NO induced N 1s signal at $399.25 \mathrm{eV}$ strongly overlaps with the signal of the nitrogen atoms in the porphyrin. 